\title{
Super-capacitive patches and their use in low-pass filters and artificial dielectrics
}

\author{
Jonathan A. Thompson $\odot$,* Giampaolo Pisano, and Carole Tucker \\ Cardiff University, School of Physics and Astronomy, The Parade, Cardiff, Wales, \\ United Kingdom
}

\begin{abstract}
The use of capacitive metal meshes consisting of square patches is well established in the design of millimeter-wave optical devices such as filters, half-wave plates, and graded-index lenses. The main property of these meshes, the capacitance, is controlled by the size of the square patch in the mesh's unit cell up to a limit imposed by the minimum feature size of the manufacturing process. We report the results of a study into the feasibility of increasing the capacitance beyond this limit by extending fingers into the adjacent unit cell. This is shown to increase the range of admittance characteristics available with the meshes and how this might be useful for the implementation of low-pass filters and artificial dielectrics is demonstrated. (c) 2021 Society of Photo-Optical Instrumentation Engineers (SPIE) [DOI: 10.1117/1.OE.60.1.017106]
\end{abstract}

Keywords: mesh filters; artificial dielectrics; millimeter waves; graded-index lens.

Paper 20201124 received Sep. 22, 2020; accepted for publication Jan. 13, 2021; published online Jan. 29, 2021.

\section{Introduction}

The use of metal mesh grids embedded in a dielectric to make millimeter-wave transmissive and reflective optical devices such as filters, half-wave plates, and graded-index (GRIN) lenses are well established ${ }^{1-3}$ and they are often seen in astronomy instrumentation. ${ }^{4-9}$ Capacitive meshes have been used to implement transmission low-pass filters, ${ }^{10}$ anti-reflection coatings, ${ }^{11}$ and GRIN lenses. ${ }^{12-14}$ The properties of these devices are limited by the range of complex admittances available from the regular arrays of isolated square patches used in the meshes. There have been a number of designs of alternate patch shapes intended for various purposes, ${ }^{15-17}$ sometimes discovered using nature-inspired algorithms ${ }^{18-23}$ with mixed results. This study concentrated on deliberately increasing the range of the prime property of a capacitive mesh; its capacitance.

The properties of a thin square patch capacitive metal mesh are governed mainly by the two physical dimensions, $g$ is the unit cell and $2 a$, the spacing between patches, as shown in Fig. 1 . The resulting admittance characteristic is often represented with an equivalent circuit, ${ }^{10,24}$ also shown in the figure. The majority of the behavior is governed by the capacitance between adjacent square patches. This is proportional to the length of the two adjacent patch sides and inversely proportional to the distance between them (in the same way as the plates in an electronic capacitor).

For a given unit cell, increasing the size of the square patch centered in it therefore increases the capacitance by simultaneously increasing the length of the adjacent sides and reducing the spacing between them. The square size can be increased until the gap is reduced to the minimum feature size supported by the manufacturing technology. A further increase in capacitance can be achieved by increasing the unit cell, but this comes with the disadvantage of reducing the frequency at which diffraction starts to occur (the frequency of the diffraction limit is inversely proportional to the unit cell).

This paper reports the results of a study into a method of increasing the capacitance further without increasing the unit cell. The proposal is to grow one or more fingers from one cell into the adjacent cell to increase the length of the adjacent sides, once the square has reached its

*Address all correspondence to Jonathan A. Thompson, thompsonja3@cardiff.ac.uk 


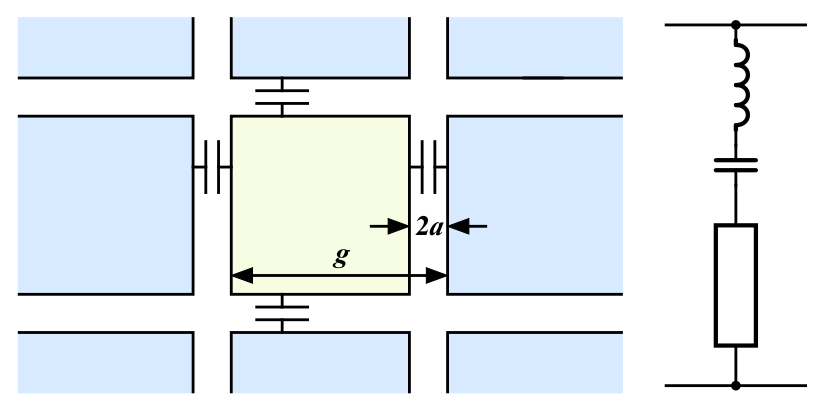

Fig. 1 A capacitive metal mesh grid consisting of square patches and an approximate equivalent circuit.

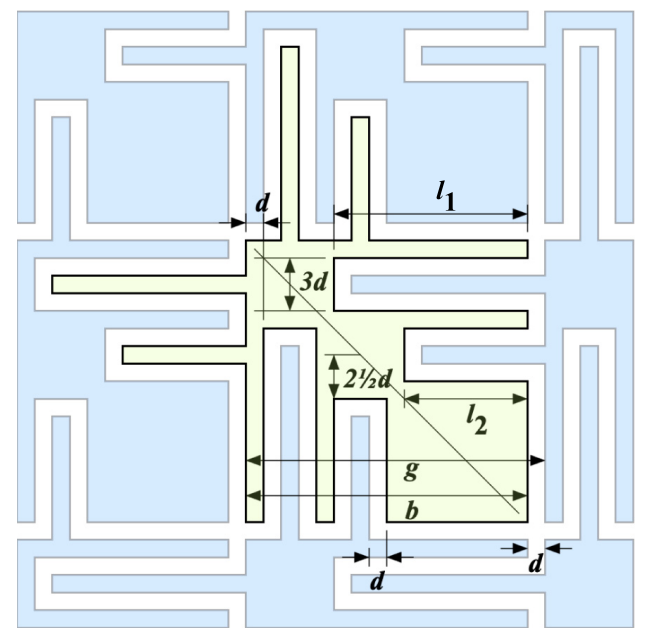

Fig. 2 The proposed pattern with fingers that can be used to increase the capacitance of a patch. Compared with a conventional square of size $b$ in the unit cell $g$, the shape increases the length of the adjacent edges and thus the capacitance. The length $d$ is the minimum feature size of the manufacturing process.

maximum size. An example with two fingers is shown in Fig. 2. The maximum length of the $n^{\prime}$ th finger being given by Eq. (1), where $d$ is the minimum feature size, $g$ is the unit cell, and $n$ is the finger number.

$$
L_{n}=g-(4 n+2) d
$$

The finger pattern can therefore be regarded as an extension to a progression of conventional capacitive square patches, as shown in Fig. 3, starting with a minimum sized conventional square ( 1 in Fig. 3) growing to the maximum size that can be fitted into the unit cell and still have a minimum feature size gap between adjacent squares (3). The first finger then starts to extend into the adjacent unit cell (4) up to its maximum size (5) where the neck between the two sockets reduces to the minimum feature size. Then a second finger extends in the same way ( 6 and 7$)$ and a third finger (8). The total number of fingers is restricted by how many can fit, given the unit cell and the minimum feature size.

One way of referring to the size of a conventional square patch is as a percentage of the unit cell covered by the square. To extend this to the finger patterns, we shall add the total length of the fingers to the size of the square and express that as a ratio of the unit cell multiplied by 100 . We shall call this number the "patch ratio." It is given by Eq. (2), where $b$ is the size of the central square, $l_{n}$ is the length of the $n$ 'th finger and $N$ is the number of fingers. 


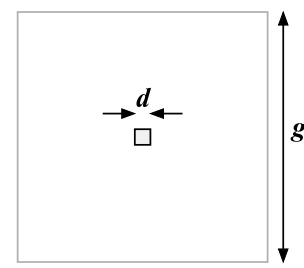

1. Minimum square.

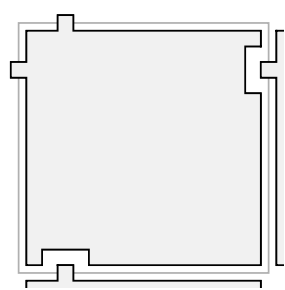

4. First finger starts.

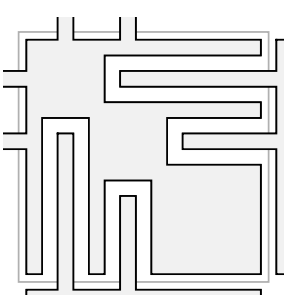

7. Second finger maximum length.

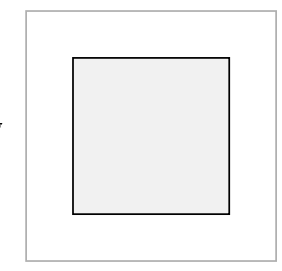

2. Growing square.

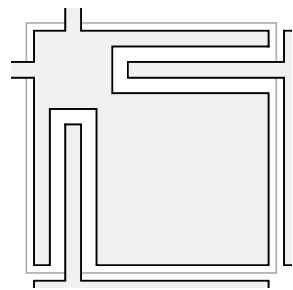

5. First finger maximum length.

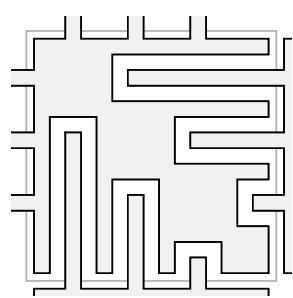

8. Third finger.

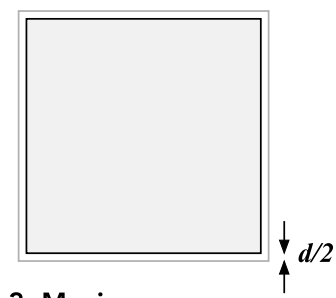

3. Maximum square.

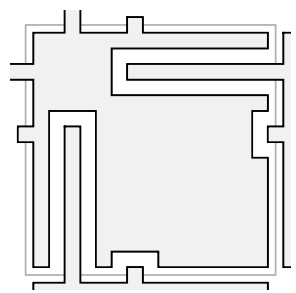

6. Second finger starts.

Fig. 3 The finger pattern (along with the precursor square patch) can be organized into a series of patterns with increasing capacitance.

$$
p=\frac{b+\sum_{n=1}^{N} l_{n}}{g} \cdot 100
$$

Consider an example unit cell of $160 \mu \mathrm{m}$ and a minimum feature size of $10 \mu \mathrm{m}$ (matching the cheap manufacturing process available to the authors), which allows patches with up to three fingers. The conventional square would start with a patch ratio of 6.25 (a $10 \mu \mathrm{m}$ square, step 1 in the figure) and rise to 93.75 (a $150 \mu \mathrm{m}$ square, step 3). A first finger of size $10 \mu \mathrm{m}$ (step 4) will be referred to as 100. The maximum sized (step 5) first finger would be 156.25 and the minimum sized second finger (step 6) would be 162.5 . The pattern with the highest capacitance (step 8 ) would have a patch ratio of 206.25 .

\section{Properties of the Super-Capacitive Patch}

The properties of the example $160 \mu \mathrm{m}$ unit cell super capacitive patch were investigated using the Ansys hight frequency structure simulator (HFSS) modeling tool. ${ }^{25}$ The modeling results of a single layer embedded in polypropylene for a selection of patch ratios are shown in Fig. 4. The patterns with fingers extend the sequence of curves nicely in the operational region (the initial downward part of the transmittance curve). The diffraction limit can also be seen at just beyond $1200 \mathrm{GHz}$, consistent with the selected unit cell. Not so useful is the appearance of a zone, marked as the resonance region in the figure, which contains a number of transmittance peaks that are very sensitive to the patch ratio.

\section{Use in Filters}

Referring again to the transmittance curves of Fig. 4, if the activity in the resonance region were to be suppressed, the super capacitive patterns could be used to implement a low-pass filter with 


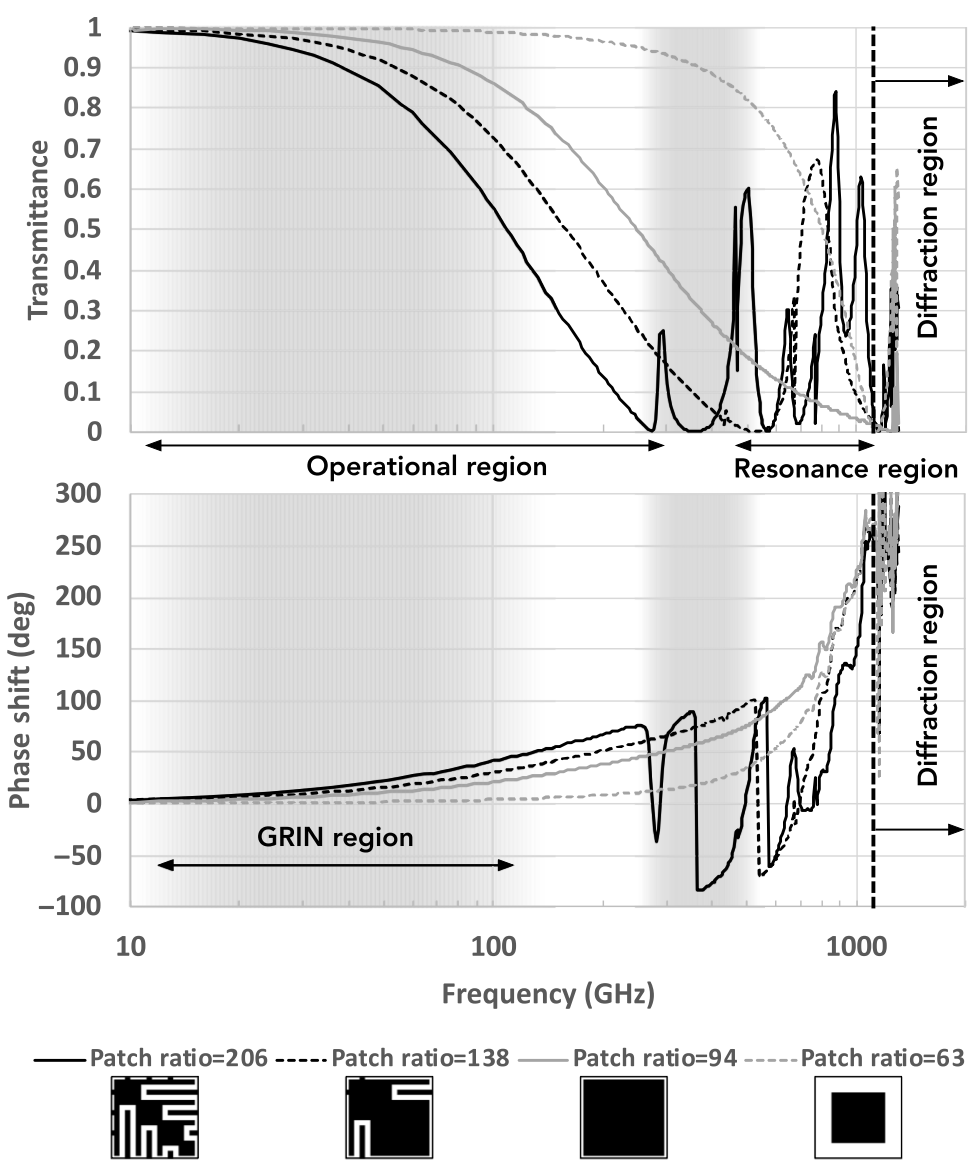

Fig. 4 Transmittance and phase shift curves for selected patterns from the sequence in a $160-\mu \mathrm{m}$ unit cell with a $10-\mu \mathrm{m}$ minimum feature size embedded in a polypropylene dielectric. The pattern pictures are of a single unit cell, so where the fingers poke into the adjacent cell, they appear as disembodied fingers in the sockets. Four regions of interest are marked. The operational region is the part of the characteristic that consists of the first smooth downward curve. The resonance region is the part that contains local peaks and troughs in the transmittance. The boundary between the operational and resonance regions depends on the patch ratio; for conventional squares, there is no resonance region. The GRIN region is the part of the characteristic most useful for creating artificial dielectrics.

a large stop band before the diffraction limit is encountered. To investigate, an existing design of low-pass filter using conventional square patches with six layers was chosen as a reference example.

A genetic algorithm and a propagation matrix modeling method using the complex admittance derived from the transmittance and phase shift ${ }^{21}$ was used to find an equivalent design using finger patterns. The minimum feature size of the finger patterns was increased to $12.5 \%$ of the unit cell (compared with the $6.25 \%$ of the patterns described in the previous sections) for the search to reduce the attenuation in the pass band, limiting the patterns to a single finger. The resulting filter used six layers all at a unit cell of $198 \mu \mathrm{m}$, layers 1 and 6 with a patch ratio of 88.75, layers 2 and 5 with a patch ratio of 111.25 , and layers 3 and 4 with a patch ratio of 118.75 . The layer spacing was $260 \mu \mathrm{m}$ and the total dielectric slab thickness was $2.04 \mathrm{~mm}$.

The two designs were then modeled using Ansys HFSS and the transmittance plots are shown in Fig. 5. Although not perfect, the principle has been demonstrated; there is a much larger stop band than the conventional example. There is still work to be done to improve the pass band and to further suppress the resonances in the stop band. 


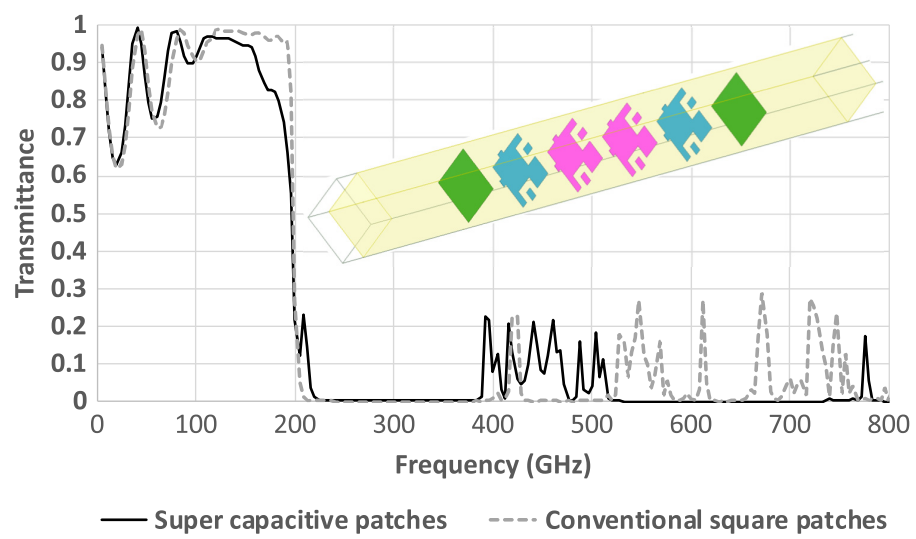

Fig. 5 The transmittance curves for the conventional square patch low-pass filter and the super capacitive patch (embedded sketch) low-pass filter. The conventional squares solution has signal in the stop band from about $400 \mathrm{GHz}$ upward due to the diffraction limit for the grids used being exceeded. In contrast, the signal in the stop band of the finger pattern is not due to diffraction, but rather to the incomplete suppression of the resonances; the diffraction limit for these grids is around $800 \mathrm{GHz}$. However, there is extra attenuation in the pass band.

\section{Use in Artificial Dielectrics}

To demonstrate the use of the finger pattern in an artificial dielectric, 10 layers of various patterns with a spacing of $25 \mu \mathrm{m}$ were modeled using Ansys HFSS. The unit cell was again $160 \mu \mathrm{m}$ and the minimum feature size $10 \mu \mathrm{m}$, allowing up to three fingers to be supported. However, we shall see that the advantage of including the third finger, which cannot grow very large, is marginal in this case. The effective refractive index was extracted from the reflection and transmission S-parameters, $S_{11}$ and $S_{21} \cdot{ }^{26,27}$

The modeling results are shown in Fig. 6. The finger pattern does increase the effective refractive index when compared to the largest conventional square. The effects become more significant beyond about $100 \mathrm{GHz}$ at the expense of increasing chromatic aberration as the refractive index varies with frequency significantly. At $200 \mathrm{GHz}$, the maximum refractive index

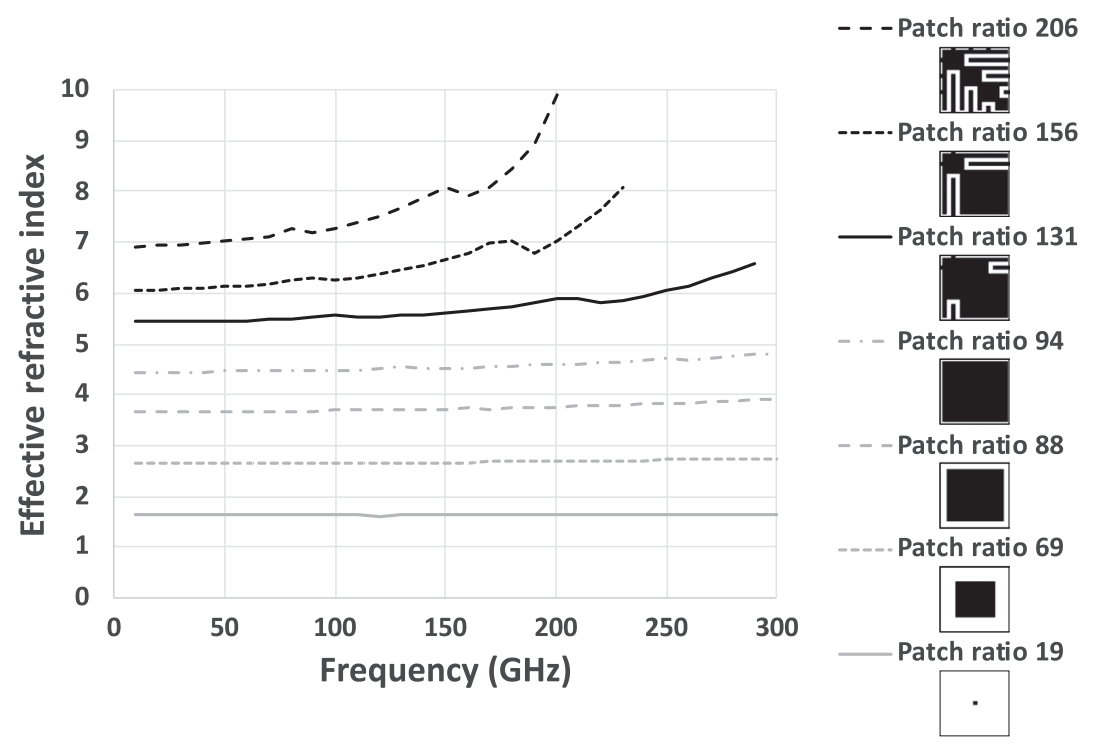

Fig. 6 The effective refractive index of 10 layers of selected finger patterns with unit cell of $160 \mu \mathrm{m}$, minimum feature size of $10 \mu \mathrm{m}$, and layer spacing $25 \mu \mathrm{m}$. Some curves stop short as the frequency reaches the cutoff and the test signal is no longer transmitted through the structure. Curves for the conventional squares are also included showing the progression. 


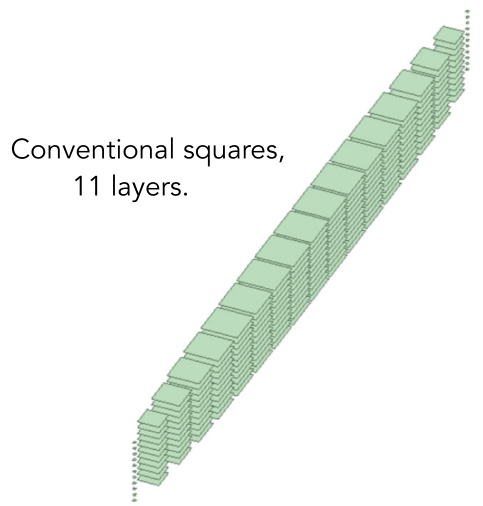

(a)

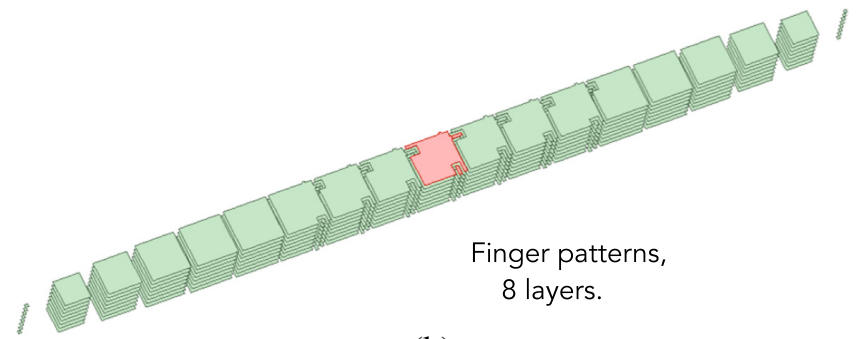

(b)

Fig. 7 The two lens design models: (a) uses eleven conventional square patch layers; (b) has eight layers and uses the finger pattern. One super capacitive patch has been highlighted to make it clearer. The models consist of a slice through each lens one unit cell wide in the $y$ direction.

achieved by the finger pattern with the unit cell and layer spacing described is 9.9 although there is attenuation and dispersion at this extreme.

To show the use of the extended range of refractive indices a pair of small graded-index lenses were designed, one restricted to conventional square patches, one allowed to use the super capacitive patches. The specification was for a 3-mm diameter lens with a focal length of $1 \mathrm{~mm}$ and an operating frequency of $200 \mathrm{GHz}$. The implementations use the same unit cell $(160 \mu \mathrm{m})$ and minimum feature size $(10 \mu \mathrm{m})$ as the data presented above. The lens specification was chosen for ease of modeling and is not necessarily useful in itself. The two designs were adjusted so that their performance was a close as possible to each other. The conventional square patch implementation required eleven layers, whereas the super capacitive patch solution was achieved in eight, see Fig. 7.

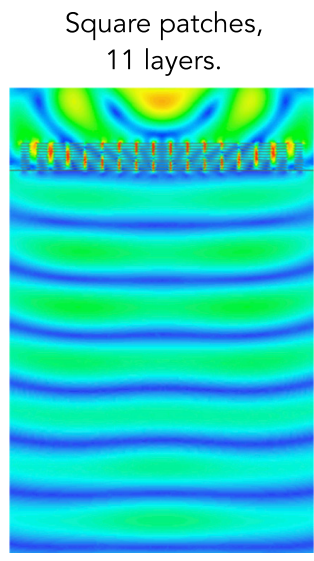

(a)

\section{Super capacitive} patches, 8 layers.

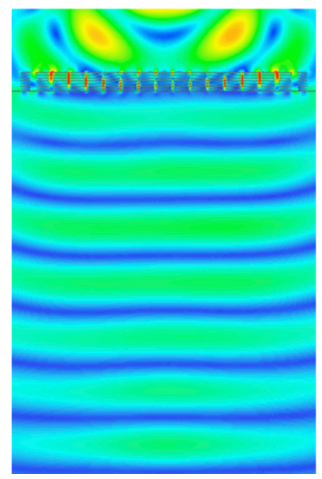

(b)

Fig. 8 The electric field magnitude of the two lens designs. This shows the very similar behavior achieved by the two implementations at the design frequency of $200 \mathrm{GHz}$. 


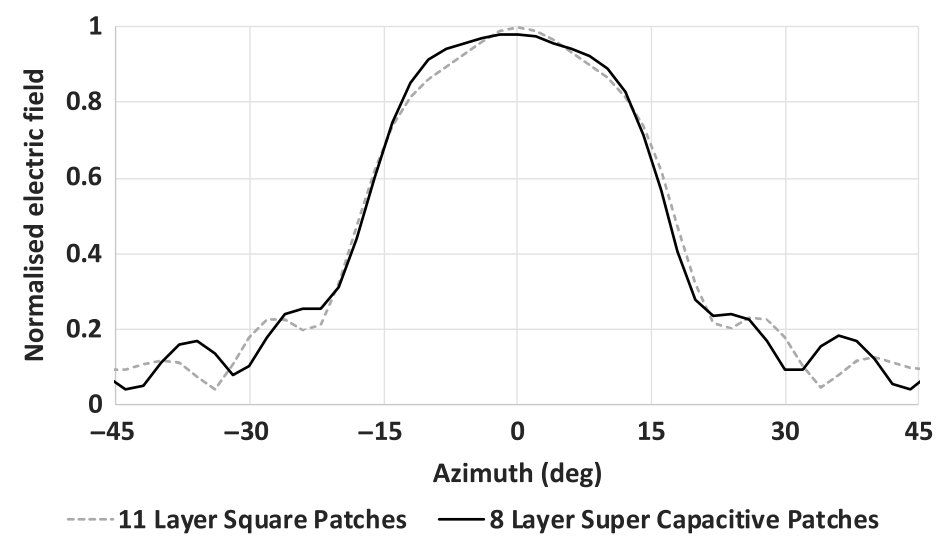

Fig. 9 The far field for the two lens designs as modeled by HFSS. The electric field magnitude is normalized so that the value at zero degrees azimuth for the eleven layer lens is 1 . Identical sources were used for the two models. The two curves are very similar, the finger pattern solution showing just slightly more attenuation at the design frequency $(-0.16 \mathrm{~dB})$. The full width at half maximum was $35.3 \mathrm{deg}$ and $34.1 \mathrm{deg}$ for conventional square patches and super capacitive patches, respectively. The behavior of the side lobes is also not too dissimilar. The first side lobes of the square patch solution are $3 \mathrm{deg}$ further away from the center. They are attenuated $-12.8 \mathrm{~dB}$ with respect to the central peak compared with super capacitive patch's $-11.7 \mathrm{~dB}$.

The designs were modeled in Ansys HFSS as a slice one unit cell thick in the $y$ direction through the lens. Periodic boundary conditions applied in the $y$ direction effectively make this a model of a cylindrical lens. A Gaussian beam source was placed at the focus point and Fig. 8 is a view of the $x, z$ plane of the lenses, showing the resultant electric field magnitudes with strikingly similar performance despite the super capacitive patch solution using three fewer layers. The far-field beam pattern is shown in Fig. 9 and again indicates how similar the performance of the two designs is.

However, there is a price to pay for the reduced number of layers. We have already discussed how the refractive index varies with frequency as the patch ratio is increased. The resulting dispersion effectively reduces the usable bandwidth of the lens as the maximum patch ratio increases. Another effect of increasing the patch ratio is the fall in the cutoff frequency that we saw in Fig. 4, placing a limit on the maximum frequency and causing extra attenuation as that frequency is approached. The example lens minimized both these effects by only using a single, small, and finger.

\section{Conclusion}

By growing fingers into the adjacent cell, it was shown that it is possible to increase the interpatch capacitance of a capacitive mesh grid beyond that possible with a conventional square patch to produce transmittance and phase-shift characteristics that can be useful. The possibility of a low-pass filter with a larger stop band before the diffraction limit was demonstrated although more work is required to optimize the pass band. The extra phase shift available was also used to create artificial dielectrics with increased refractive indices, which were used to reduce the number of layers required by an example GRIN lens. More work is required to investigate their use in more realistic lens designs.

The manufacture and verification of devices using super capacitive patches remains as further work due to the Covid-19 pandemic bringing work to a halt for the foreseeable future. The group at Cardiff has extensive experience with the modeling and construction of metal mesh millimeter-wave devices such as filters, half-wave plates, ${ }^{3,28}$ Toraldo pupils, ${ }^{29}$ magnetic mirrors, ${ }^{30}$ and lenses, ${ }^{14,31}$ so there is confidence that real-world implementations will match well. 


\section{Acknowledgments}

We acknowledge the support of the Supercomputing Wales project, which is part-funded by the European Regional Development Fund (ERDF) via Welsh Government. The authors declare no conflicts of interest.

\section{References}

1. B. A. Munk, Frequency Selective Surfaces: Theory and Design, John Wiley \& Sons, Inc., Hoboken, NJ (2000).

2. G. Pisano et al., "Metal mesh based metamaterials for millimetre wave and $\mathrm{THz}$ astronomy applications," in 8th UK, Europe, China Millimeter Waves and THz Technol. Workshop, pp. 1-4 (2015).

3. G. Pisano et al., "Multi-octave metamaterial reflective half-wave plate for millimeter and sub-millimeter wave applications," Appl. Opt. 55, 10255-10262 (2016).

4. M. J. Griffin et al., "The Herschel-SPIRE instrument and its in-flight performance," Astron. Astrophys. 518, L3 (2010).

5. D. S. Swetz et al., "Overview of the Atacama cosmology telescope: receiver, instrumentation and telescope systems," Astrophys. J. Suppl. Ser. 194, 41 (2011).

6. R. J. Thornton et al., "The Atacama cosmology telescope: the polarization-sensitive Actpol instrument," Astrophys. J. Suppl. Ser. 227, 21 (2016).

7. M. H. Abitbol et al., "CMB-S4 technology book, first edition," 2017, https://arxiv.org/abs/ 1706.02464 (accessed 24 January 2021).

8. L. M. Fissel et al., "The balloon-borne large-aperture submillimeter telescope for polarimetry: BLAST-Pol," Proc. SPIE 7741, 77410E (2010).

9. J.-P. Bernard et al., "PILOT: a balloon-borne experiment to measure the polarized FIR emission of dust grains in the interstellar medium," Exp. Astron. 42, 199-227 (2016).

10. P. A. R. Ade et al., "A review of metal mesh filters," Proc. SPIE 6275, 62750U (2006).

11. J. Zhang et al., "New artificial dielectric metamaterial and its application as a terahertz antireflection coating," Appl. Opt. 48, 6635-6642 (2009).

12. V. N. Nguyen, S. H. Yonak, and D. R. Smith, "Millimeter-wave artificial dielectric gradient index lenses," in 3rd Eur. Conf. Antennas and Propag., pp. 1886-1890 (2009).

13. G. Savini, P. A. R. Ade, and J. Zhang, "A new artificial material approach for flat $\mathrm{THz}$ frequency lenses," Opt. Express 20, 25766-25773 (2012).

14. G. Pisano et al., "Development of flat silicon-based mesh lens arrays for millimeter and sub-millimeter wave astronomy," J. Low Temp. Phys. 199, 923-934 (2020).

15. B. Monacelli et al., "Infrared frequency selective surface based on circuit-analog square loop design," IEEE Trans. Antennas Propag. 53, 745-752 (2005).

16. D. Schurig et al., "Metamaterial electromagnetic cloak at microwave frequencies," Science 314, 977-980 (2006).

17. I. Mohamed et al., "Dog bone triplet metamaterial wave plate," in PIERS Kuala Lumpur Proc., The Electromagnetics Academy, Kuala Lumpur, pp. 1756-1760 (2012).

18. R. L. Haupt and D. H. Werner, Genetic Algorithms in Electromagnetics, John Wiley \& Sons, Hoboken, NJ (2007).

19. Y. Ge and K. P. Esselle, "GA/FDTD technique for the design and optimisation of periodic metamaterials," Antennas Propag. IET Microwaves 1, 158-164 (2007).

20. B. Choudhury, S. Bisoyi, and R. M. Jha, "Emerging trends in soft computing techniques for metamaterial design and optimization," Comput. Mater. Continua 31(3), 201-228 (2012).

21. J. A. Thompson and G. Pisano, "Use of evolutionary computing algorithms in the design of millimetre-wave metamaterial devices," Proc. SPIE 10708, 107083H (2018).

22. S. D. Campbell et al., "Review of numerical optimization techniques for meta-device design [Invited]," Opt. Mater. Express 9, 1842-1863 (2019).

23. J. A. Thompson, G. Pisano, and C. Tucker, "Mesh low-pass filters for millimeter-wave applications: is the square capacitive shape optimal?" J. Astron. Telesc. Instrum. Syst. 6, 036003 (2020). 
Thompson, Pisano, and Tucker: Super-capacitive patches and their use in low-pass filters...

24. R. Ulrich, "Far-infrared properties of metallic mesh and its complementary structure," Infrared Phys. 7, 37-55 (1967).

25. "ANSYS HFSS: high frequency electromagnetic field simulation software," https://www .ansys.com/en-gb/products/electronics/ansys-hfss (accessed 18 December 2019).

26. A. M. Nicolson and G. F. Ross, "Measurement of the intrinsic properties of materials by time-domain techniques," IEEE Trans. Instrum. Meas. 19, 377-382 (1970).

27. X. Chen et al., "Robust method to retrieve the constitutive effective parameters of metamaterials," Phys. Rev. E 70, 016608 (2004).

28. G. Pisano et al., "Development of large radii half-wave plates for CMB satellite missions," Proc. SPIE 9153, 915317 (2014).

29. G. Pisano et al., "Metamaterial-based Toraldo pupils for super-resolution at millimetre wavelengths," Proc. SPIE 10708, $107080 \mathrm{G}$ (2018).

30. G. Pisano, P. A. R. Ade, and C. Tucker, "Experimental realization of an achromatic magnetic mirror based on metamaterials," Appl. Opt. 55, 4814-4819 (2016).

31. G. Pisano et al., "Development of large-diameter flat mesh-lenses for millimetre wave instrumentation," Proc. SPIE 10708, 107080D (2018).

Jonathan A. Thompson is a postgraduate student working toward a PhD in the School of Physics and Astronomy at Cardiff University, UK. His interests are in the use of meta-materials for the implementation of filters and lenses in the field of millimeter-wave astronomy.

Giampaolo Pisano is a reader in the School of Physics and Astronomy at Cardiff University, UK. His primary interest is new instrumentation focused on the detection of B-modes in the cosmic microwave background. He has developed high-performance RF devices such as phase shifters, orthomode transducers, polarizers, and polarization modulators along with novel quasioptical components based on metamaterials such as mesh half-wave plates, mesh lenses, and magnetic mirrors.

Carole Tucker is a professor in the School of Physics and Astronomy at Cardiff University, UK. Her particular expertise lies with the quasi-optical filter technology for which Cardiff is the sole-provider worldwide. She has interests in the fields of FIR astronomical instrumentation for photometric and spectroscopic studies, quasi optics and metamaterials for FIR Astronomy and $\mathrm{THz}$ applications, IR to $\mathrm{THz}$ spectroscopy of optical components and materials, and cryogenic instrumentation. 